\title{
The evolution of activity in galaxies from $z=0.45$ to the present time
}

\author{
Grażyna Stasińska ${ }^{1}$, M. V. Costa-Duarte ${ }^{1,2}$ and N. Vale Asari ${ }^{3}$ \\ ${ }^{1}$ LUTH, Observatoire de Paris, CNRS, Université Paris Diderot, \\ Place Jules Janssen, F-92190 Meudon \\ email: grazyna.stasinska@obspm.fr \\ ${ }^{2}$ Instituto de Astronomia, Geofísica e Ciências Atmosféricas, Universidade de São Paulo, \\ São Paulo, Brazil \\ email: mvcduarte@astro.iag.usp.br \\ ${ }^{3}$ Departamento de Física - CFM - Universidade Federal de Santa Catarina, \\ Florianópolis, SC, Brazil \\ email: natalia@astro.ufsc.br
}

\begin{abstract}
We use spectra from the SDSS to study the signatures of nuclear activity over cosmic time, dividing the samples in redshift and mass bins, and using various diagnostic diagrams. A redshift evolution clearly emerges from the analysis of our diagrams.
\end{abstract}

Keywords. surveys, galaxies: general, galaxies: active

\section{Introduction}

Studying the interplay between star forming, AGN and passive phases of galaxy evolution requires:

- A wide database of adequate galaxy spectra.

- Properly defined criteria for these different phases of galaxy evolution.

- Binning the sample into stellar mass $\left(M_{\star}\right)$ and redshift $(z)$ bins to adequately deal with the problem of downsizing (Cowie et al. 1996) and completeness.

\section{The binned SDSS/DR7 galaxy sample}

- We extracted our database from the Sloan Digital Sky Survey (SDSS/DR7, Abazajian et al. 2009) main galaxy sample, applying the following restrictions: redshift $>0.002$ and $z$-band aperture covering factor $>20 \%$ (to reduce aperture effects and avoid intragalactic sources). Additional restrictions were applied depending on the considered properties (see below)

- The current total stellar mass $M_{\star}$ and the measurements of the H $\alpha$, [NII]6584, [OIII] 5007 and $\mathrm{H} \beta$ emission lines were taken from the STARLIGHT database (http://casjobs.starlight.ufsc.br/casjobs/).

- The database was divided in $M_{\star}$ bins of 0.5 dex between $9<\log M_{\star} / \mathrm{M}_{\odot}<12$ and in $z$ bins of 0.05 from 0.05 to 0.45 . Bins corresponding $\log M_{\star} \mathrm{M}_{\odot}<9$ and $\log M_{\star} \mathrm{M}_{\odot}>12$ are also shown but they cover a wider range of stellar masses. 


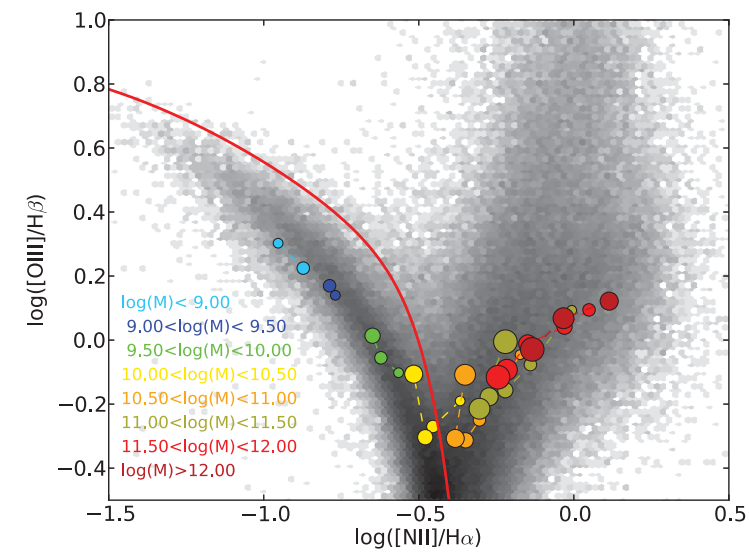

Figure 1. The BPT diagram for galaxies that have a signal-to-noise ratio of at least 3 in the four relevant lines. Circles show the position of the average values of $[\mathrm{OIII}] / \mathrm{H} \beta$ vs $[\mathrm{NII}] / \mathrm{H} \alpha$ for each $\left(M_{\star}, z\right)$ bin. Larger circles represent higher redshifts. Different colours represent different $M_{\star}$ bins, blue for the smallest $M_{\star}$ and red for the largest ones. The curve represents the S06 divisory line. The background density map represents the entire SDSS/DR7 galaxy sample from $0.05<z<0.1$.

\section{The BPT and WHAN diagrams}

\subsection{The BPT diagram}

To distinguish between $\mathrm{SF}$ galaxies and AGN hosts, the $[\mathrm{OIII}] / \mathrm{H} \beta$ versus $[\mathrm{NII}] / \mathrm{H} \alpha$ diagram (Baldwin et al. 1981, BPT) is generally used. Since the study by Kauffmann et al. (2003, K03) it is common practise to consider galaxies as star-forming when they lie below the empirical K03 line, AGN hosts when they lie above the "extreme starburst line" of Kewley et al. (2001, K01) line, and "composite" when they are found in-between. However Stasińska et al. (2006, S06) and Dopita et al. (2013) have produced a more appropriate theoretical line to separate pure $\mathrm{SF}$ galaxies from the rest.

- When plotting the average values of $[\mathrm{OIII}] / \mathrm{H} \beta$ versus $[\mathrm{NII}] / \mathrm{H} \alpha$ it appears that the 3 lowest $M_{\star}$ bins are all entirely in the pure SF region and that the metallicity of the interstellar medium (as indicated by $[\mathrm{NII}] / \mathrm{H} \alpha$ ) increases with time.

- The higher $M_{\star}$ bins are located in the right wing. Taking the BPT diagram as a perfect diagnostic for the presence of AGNs, one would erroneously infer that the importance of AGN versus star formation increases with time for these $M_{\star}$ bins, since the points tend to go to the right as redshift decreases.

\subsection{The WHAN diagram}

However, as shown by Stasińska et al. (2008) and further discussed by Cid Fernandes et al. (2011), retired galaxies (i.e., emission-line galaxies that have stopped forming stars and are ionized by hot low-mass evolved stars - HOLMES) have the same location in the BPT diagram as galaxies hosting weak AGNs.

- The WHAN diagram (Cid Fernandes et al. 2011) allows one to distinguish between pure SF galaxies, AGN hosts (strong and weak) and passive galaxies (i.e. retired galaxies and lineless galaxies).

- The highest $M_{\star}$ bins clearly enter the region of passive galaxies as redshift decreases. 


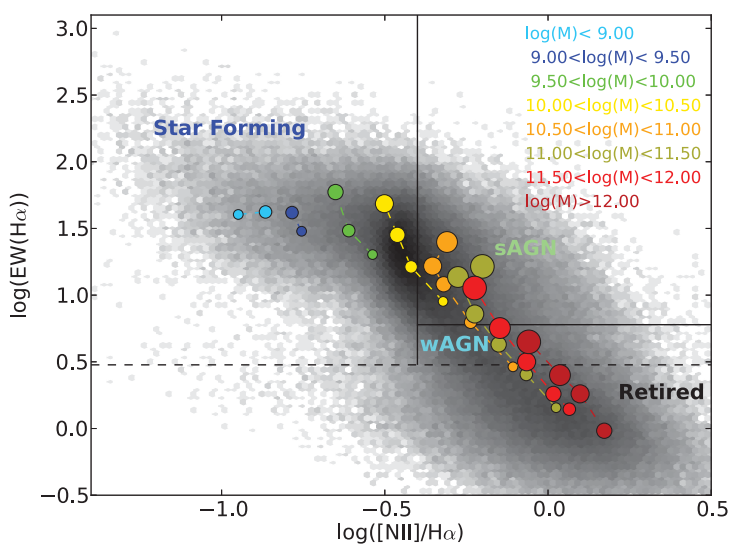

Figure 2. The WHAN diagram. The circles represent the position of the average values of $\mathrm{EW}(\mathrm{H} \alpha)$ versus $[\mathrm{NII}] / \mathrm{H} \alpha$ for each $\left(M_{\star}, z\right)$ bin in our database, excluding those galaxies whose spectra have "bad" pixels in the $\mathrm{H} \alpha$ or [NII] region.

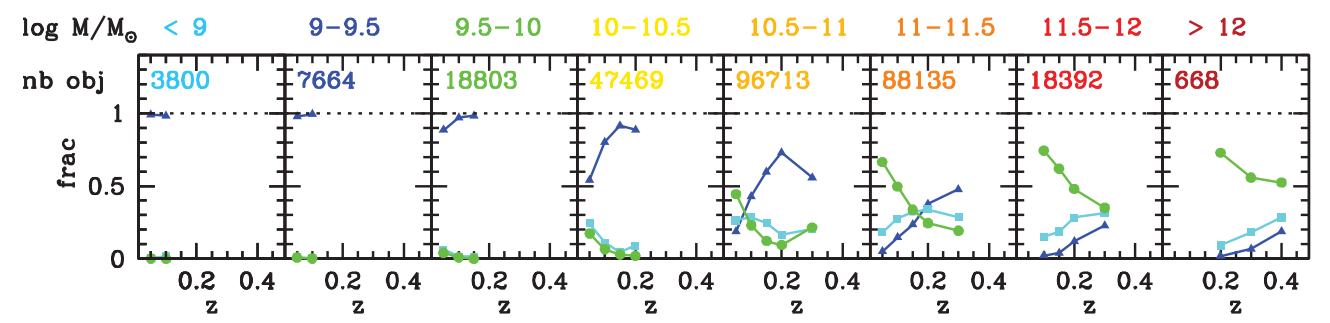

Figure 3. Fraction of galaxies of different spectral types as a function of redshift for the different mass bins as obtained from the canonical use of the BPT diagram using the K01 and K03 divisory lines. Blue triangles: SF; green circles: AGN, and cyan squares: composite.

\section{A detailed census of galaxy types in $\left(M_{\star}, z\right)$ bins}

While discussing how average properties of galaxies change with $M_{\star}$ or $z$, one must nor forget to also examine their dispersion. In the $\left(M_{\star}, z\right)$ bins, some galaxies can be pure SF ones, while others may contain an AGN, and many of them may have no emission lines at all.

\subsection{According to the classical use of the BPT diagram}

- Low $M_{\star}$ bins present a predominance of SF galaxies. As $M_{\star}$ increases the presence of an AGN host becomes more common and becomes dominant at the highest $M_{\star}$.

- In the highest $M_{\star}$ bins "composite" galaxies behave similarly to SF galaxies, while they behave similarly to AGN galaxies for $\log M_{\star} / \mathrm{M}_{\odot}<11$.

- The proportion of SF galaxies decreases as redshift decreases while that of AGN hosts increases with decreasing redshift. For $\log M_{\star} \mathrm{M}_{\odot}<10.5$ the population of galaxies entering the BPT diagram and classified in the canonical way as SF largely dominates at any redshift.

\subsection{According to the WHAN diagram}

The WHAN diagram allows one to distinguish between weak AGNs and retired galaxies (erroneously counted as AGN hosts or "composite" when using the BPT diagram in a classical way). This permits a true census of galaxies of various spectral types in the local Universe. Note that, like Cid Fernandes et al. (2011) we distinguish between pure SF 


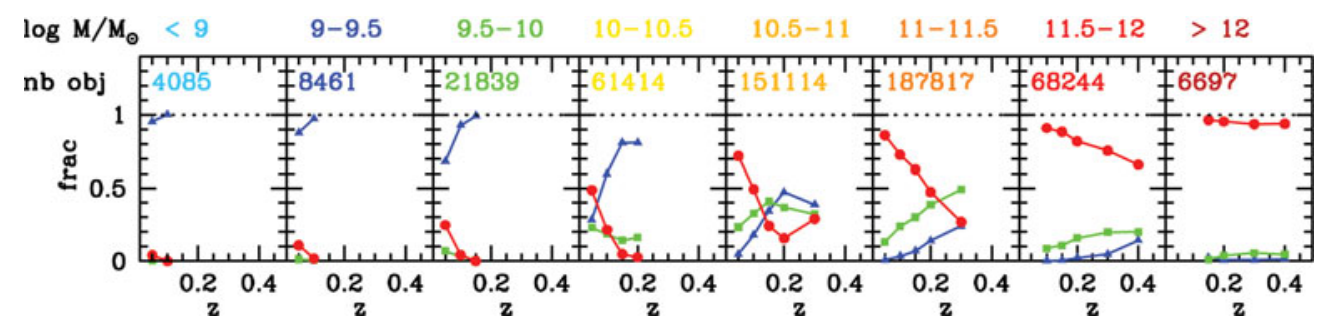

Figure 4. Fraction of SF galaxies (blue triangles), AGN hosts (green squares) and passive retired and lineless - galaxies (red circles) found from the WHAN diagram as a function of $z$.

galaxies and galaxies showing at least a trace of an AGN contribution (so our definition of SF objects is not identical to that of Kauffmann et al. 2003). We see that:

- Low $M_{\star}$ bins present a predominance of SF galaxies while high $M_{\star}$ bins present a predominance of passive galaxies. AGN hosts never represent the dominant fraction if all galaxies are taken together.

- The fraction of passive galaxies increases with decreasing $z$ indicating a decrease of star formation with time. The highest $M_{\star}$ bins, however, are almost entirely populated by passive galaxies at all the sampled redhifts, indicating that the bulk of star formation in them occured over 4 Gyr ago (corresponding to $z=0.4$ ).

\section{Summary and conclusions}

- Because of downsizing, the study of the evolution of SF galaxies and AGN hosts needs to be done in stellar mass bins.

- Retired galaxies cannot be distinguished from weak AGNs (LINER-type galaxies) in the BPT diagram. Differentiation can be done using the WHAN diagram.

- Studies dealing with the redshift evolution of galaxies cannot ignore the existence of lineless galaxies, and thus cannot be based on emission-line diagnostics only.

- The BPT and WHAN diagram lead to very different views of the evolution of galaxy spectral types in the local Universe.

\section{References}

Abazajian, K. N., et al., 2009, ApJS, 182, 543

Baldwin, J. A., Phillips, M. M., \& Terlevich, R., 1981, PASP, 93, 5

Cid Fernandes, R., Stasińska, G., Mateus, A., \& Vale Asari, N., 2011, MNRAS, 413, 1687

Cowie, L. L., Songaila, A., Hu, E. M., \& Cohen, J. G., 1996, AJ, 112, 839

Dopita, M. A., et al. , 2013, ApJS, 208, 10

Kauffmann, G., et al., 2003, MNRAS, 346, 1055

Kewley, L. J., Dopita, M. A., Sutherland, R. S., Heisler, C. A., \& Trevena, J., 2001, ApJ, 556,121

Stasińska, G., et al., 2008, MNRAS, 391, L29 